\title{
Model-based compensation of rate-dependent hysteresis in a piezoresistive strain sensor
}

\author{
Alberto Oliveri, Member, IEEE, Martina Maselli, Matteo Lodi, Student Member, IEEE, \\ Marco Storace, Senior Member, IEEE, and Matteo Cianchetti, Member, IEEE
}

\begin{abstract}
This paper is concerned with modeling of smart textiles, aimed at compensating their intrinsic nonlinearities. In particular, a new model is proposed to compensate for hysteresis and relaxation in strain sensors made of Electrolycra. These sensors are increasingly employed in emerging areas such as wearable electronics and soft robotics for their simple transduction mechanism and low cost. However, being intrinsically nonlinear, the signals measured from these devices need some processing, in order to increase their sensing accuracy. Here, we propose a new model for the compensation of the main distortions intrinsic to these soft sensors, which are mainly caused by hysteresis and relaxation, whose combined effect produces rate-dependent hysteresis. The model capabilities are tested on experimental data measured on Electrolycra. The comparisons with the results obtained with two different models witness the good behavior of the proposed model.
\end{abstract}

Index Terms-Conductive textile, piezoresistive strain sensor, rate-dependent hysteresis, hysteresis compensation.

\section{INTRODUCTION}

$\mathbf{S}$ TRAIN gauge sensors have been widely used in robotics [1], [2] for their mechanical and electrical simplicity, compact form and cost effectiveness, providing relatively high accuracy for measuring small strains [3]. However, their measurement range is limited by the material of conventional strain gauges, thus making them unsuitable for soft structures that are subject to large deformations. Recent interest in wearable electronics [4], human/machine interfaces [5], soft exosuits [6], and soft robots [7], [8], led to the development of a new class of soft strain sensors that can be both highly conformable and extensible.

A popular approach to create flexible and highly stretchable strain sensors is to mix conductive additives to soft silicone rubber materials [9]. These sensors can support very large

Manuscript received July 25, 2018; revised November 8, 2018; accepted November 19, 2018. This work was supported in part by the University of Genoa and by the European Commission through the Hybrid Heart Project under Grant 767195.

Alberto Oliveri, Matteo Lodi and Marco Storace are with the Department of Electrical, Electronic, Telecommunications Engineering and Naval Architecture (DITEN), University of Genoa, Via Opera Pia, 11A, 16145 - Genova, Italy - alberto.oliveri; matteo.lodi; marco.storace@unige.it.

Martina Maselli and Matteo Cianchetti are with The BioRobotics Institute, Scuola Superiore Sant'Anna, Viale Rinaldo Piaggio, 34, 56025 - Pontedera (PI), Italy - m.maselli; matteo.cianchetti@santannapisa.it. elongations and typically have relatively high gauge factors [10]. However, the resistance variation is dominated by complex effects such as quantum tunnelling [11], that are difficult to master. This limitation has motivated the development of highly stretchable sensors composed of hyperelastic elastomers with embedded microchannels filled with a conductive liquid, such as eutectic gallium-indium (eGaIn) [12]. Although these sensors offer relatively high accuracy, reliability for measuring large strains, and less hysteresis, the conductive liquid metal material is expensive and the fabrication process requires manual skills or custom printing hardware to obtain a correct injection of the liquid into the intricate channels of the soft body [13].

Another approach is the use of capacitive-type sensors [14], [15], which typically employ a highly compliant dielectric layer sandwiched between two stretchable electrodes. Tensile strain brings the electrodes closer and results in an increase of capacitance [16]. However, design and fabrication require a complex tuning of many material properties, such as dielectric constant, dielectric loss, and mechanical properties [7].

Sensing solutions based on smart textiles can provide a valid alternative as soft strain sensors. Indeed, textile-based solutions have several advantages: low cost, lightness, flexibility and capacity to adapt to different body structures [17]. Smart textiles are fabrics that can sense and react to mechanical, thermal, chemical, magnetic and electric stimuli, by means of extrinsic and intrinsic modifications [18].

Piezoresistive sensor technology [19] is a common method for creating textile-based strain sensors along with capacitive [20], inductive and impedance sensors [21]. Usually, piezoresistive strain sensors exploit the electrical resistance change due to an applied mechanical stretching, but they present remarkable electro-mechanical hysteresis and relaxation dynamics. This is known as a major drawback of resistance-type sensors, which can limit severely the applicability of these materials as sensors. Friction force and structural changes in the material are reported as the main causes of this undesirable behavior [22]. Several methods have been proposed to reduce the impacts of these factors [23], [24], but compensation through mathematical modeling is unavoidable to improve the stretch sensing accuracy. Hysteresis compensators based on a modified dynamic Preisach model [25] or on a semilinear Duhen model [26] were applied on strain sensors made of conductive polymer nanocomposites, but with limited results in terms of performance and generalizability. More in general, methods to compensate for hysteresis and relaxation effects 
are available in literature: the interested reader can refer to [27] for a survey about pros and cons of different models and compensators and to [28] for a survey focused on nanopositioning applications, where relaxation (known as creep in this context) plays an important role.

We present a thorough experimental investigation probing the electromechanical response of a conductive textile subjected to cyclic loading and we propose a new model to compensate for its intrinsic nonlinearities, thus improving its sensing accuracy and paving the way to precise strain measurement in soft highly stretchable sensors. The new model is a variant of a power-law (PL) model recently proposed [29] to reproduce hysteresis and creep in piezoelectric actuators (PEAs), which are largely used for micro- and nanopositioning applications [30], [31]. In this work the PL model is properly modified in order to compensate for the ratedependent hysteresis of the conductive textile. The model can be exploited to accurately estimate the strain of the textile, given the measurement of its electrical resistance and is valid at different strain rates. The model parameters are identified on a set of experimental data and its accuracy is validated on a different set. We also benchmarked the results obtained with our model against both the MPI model and an algebraic model that relates strain and resistance through a third-order polynomial.

The rest of this paper is organized as follows. Section II describes experimental setup, measurement protocol and proposed model. Compensation results are presented and discussed in Sec. III, before drawing some conclusions in Sec. IV.

\section{MATERIALS AND METHOdS}

\section{A. Experimental setup}

Among the available possibilities on the market, the Electrolycra (Mindsets Ltd, United Kingdom) has been selected for this study. Previous works have adopted Electrolycra as a strain sensor in several applications such as the sensorisation of continuum soft robots for reconstructing their spatial configuration [8], or to reveal bending and force in a soft body [32], or as a wearable goniometer [33], [34].

Electrolycra looks and feels like ordinary lycra but it is highly conductive because it is weaved with silver plated $76 \%$ nylon $24 \%$ elastic fibers [8]. Its conductivity depends on how tightly it is stretched; if it is stretched, its resistance $R$ increases.

In particular, we are interested in the relationship between strain $S=100 \frac{L-L_{0}}{L_{0}} \%$ and resistance $R$, where $L$ is the current sample length, whereas $L_{0}$ is the sample resting length. Electrolycra can be stretched beyond its initial length, with better performances after pre-stretching of $200 \%$ [33]. Moreover, this material has a preferential direction along which resistance significantly changes and a major strain is possible, with a working range of strain $S$ from $0 \%$ to $50 \%$. Additional tests were performed for the present work to better understand Electrolycra electromechanical behavior and to validate the developed model with new experimental datasets. The electromechanical tests were performed at room temperature by using the Instron material testing machine (model 4464, Instron Inc., Norwood, MA), which allows imposing a desired strain to the textile. Each end of the sample was fixed mechanically using clamps with copper sheets and welded wires. These wires were connected to a data acquisition board (DAQ, National Instruments ${ }^{\circledR}$ ) to measure the electrical resistance values, which were simultaneously recorded for different strains with a sampling frequency of $10 \mathrm{~Hz}$. The resistance value $R$, in particular, is measured by means of a voltage divider, composed of the Electrolycra and a resistor with resistance $R_{1}=10.5 \Omega$ connected in series, with an applied voltage $V_{i n}=3.2 \mathrm{~V}$. The resistance of the Electrolycra is therefore computed as $R=R_{1} \frac{V_{i n}-V_{R 1}}{V_{R 1}}$, being $V_{R 1}$ the voltage across resistor $R_{1}$.

Due to material preferential direction [8], [33], uniaxial cyclic strain tests have been conducted along this direction on a specimen $20 \mathrm{~mm}$ wide and with length $L_{0}=100 \mathrm{~mm}$ along the preferential direction. The dimensions of the specimen were chosen arbitrarily, as the sensor behavior depends only slightly on the length-width ratio [33].

\section{B. Experimental protocol}

We collected 13 datasets of applied strain $S$ and corresponding resistance $R$, belonging to three different classes:

- Different strain patterns (datasets SP1, SP2 and SP3): three strain profiles composed of pieces of triangular waves with 5 periods each (see Fig. 1). The sample is stretched at a constant rate of $50 \mathrm{~mm} / \mathrm{min}$. In SP1 each piece is characterized by a different offset $(10 \%, 20 \%$, $20 \%$ and $30 \%)$ and amplitude $(20 \%, 20 \%, 30 \%$ and $20 \%)$; in SP2 the offset is constant (10\%) and only the amplitude changes $(10 \%, 20 \%, 30 \%$ and $40 \%)$; in SP3 the amplitude is constant $(20 \%)$ and the offset changes $(10 \%, 20 \%$ and $30 \%)$.

- Different strain rate (datasets from SR1 to SR9): nine strain profiles composed of triangular waves. For each dataset, the sample is stretched at a different rate, from $20 \mathrm{~mm} / \mathrm{min}$ (SR1) to $100 \mathrm{~mm} / \mathrm{min}$ (SR9), with an increasing step of $10 \mathrm{~mm} / \mathrm{min}$. Fig. 2 shows the time evolution of strain and resistance (left panels) and the relationship between $S$ and $R$ (right panel) for datasets SR1 and SR9. The other datasets, not shown to improve the visibility of the graph, are distributed among the curves of datasets SR1 and SR9. Notice that this relationship is hysteretic and, as the strain rate increases, the loop rotates counterclockwise around its low-left corner. Moreover, each loop is traveled clockwise, as marked by the arrows in Fig. 2. These datasets allow to evaluate the influence of the strain rate on the sensor characteristics.

- Relaxation behaviour (dataset RB1): in this case the sample was stretched at a speed of $1000 \mathrm{~mm} / \mathrm{min}$, and held at increasing values of strain $(10 \%, 20 \%, 30 \%$, $40 \%$, and $50 \%$ ) for 30 seconds (see Fig. 3). Notice the temporal decrease (relaxation) of the resistance in response to a constant strain applied to the sensor. This effect is particularly visible when the textile is stretched (black ellipse), whereas it is negligible when the textile is relaxed (gray ellipse). 


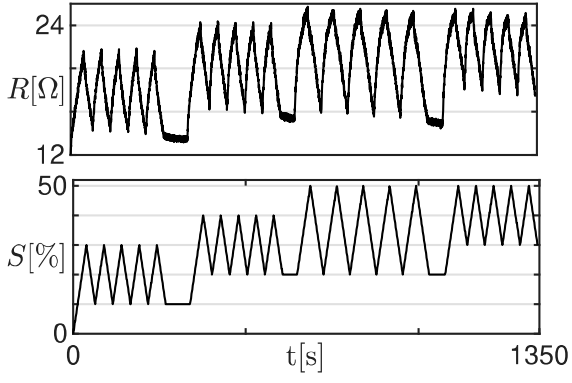

(a)

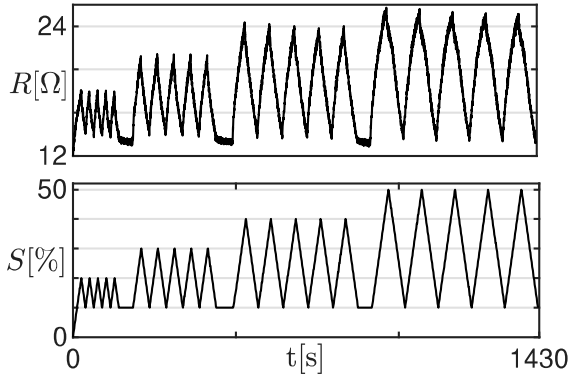

(b)

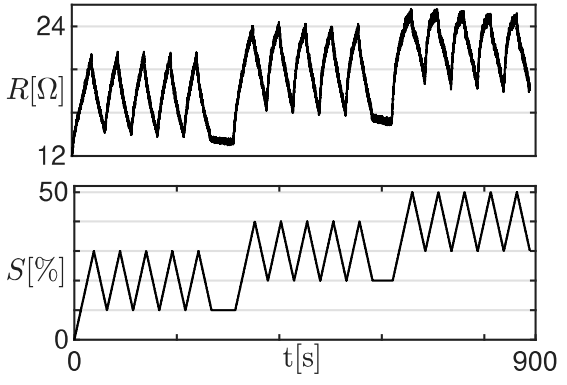

(c)

Fig. 1. Measurements of applied strain (bottom panels) and corresponding resistance (top panels) as a function of time, for datasets SP1 (a), SP2 (b) and SP3 (c).
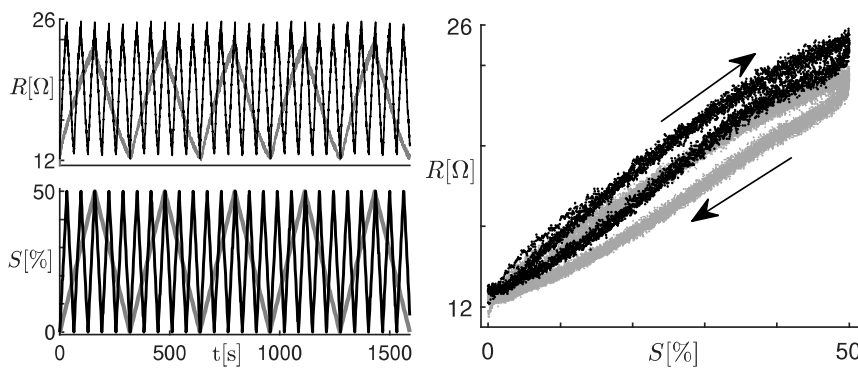

Fig. 2. Measurements of applied strain (left bottom panel) and corresponding resistance (left top panel) as a function of time for datasets SR1 (gray curves) and SR9 (black curves). The right panel shows the same measurements in the $(S, R)$ plane.

\section{Hysteresis model}

In order to accurately employ the Electrolycra as a strain sensor, $S$ must be estimated based on the measurement of the resistance $R$. An inverse model (simply referred to as model, in the following) able to reproduce both the hysteresis characteristic and the relaxation dynamics, is therefore necessary, which takes in input the measured resistance $R$ and provides an estimation $\hat{S}$ of the corresponding strain $S$. To estimate the strain, the model must be connected in cascade to the sensor, as shown in Fig. 4.

The model must be able to compensate for the typical nonlinear behaviors of Electrolycra:

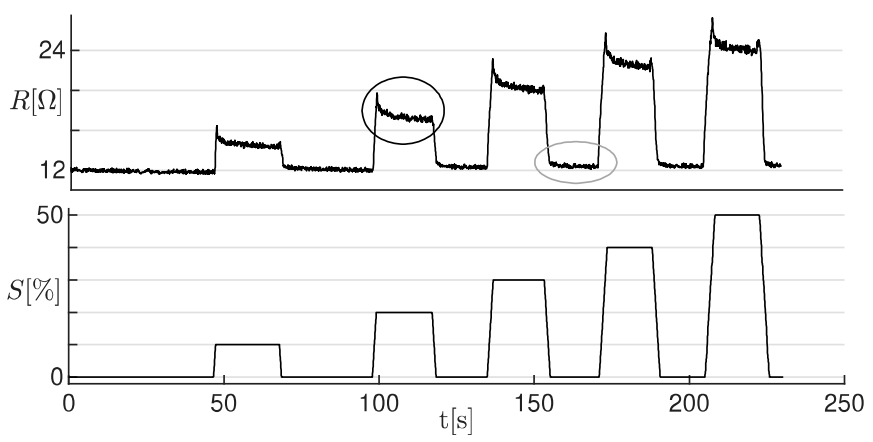

Fig. 3. Measurements of applied strain (bottom panel) and corresponding resistance (top panel) as a function of time for datasets RB1. Notice (see the black ellipse) the relaxing behavior of the resistance in response to a constant strain, when the textile is stretched.



Fig. 4. Block scheme showing how to connect the model to the Electrolycra in order to estimate the strain $S$ based on the measurement of the resistance $R$.

1) the relationship between Electrolycra input $S$ and output $R$ is hysteretic, and the hysteresis loop is traveled clockwise;

2) the hysteresis loop rotates counterclockwise around its lower-left corner as the strain rate increases;

3 ) in the presence of a constant strain, Electrolycra resistance slowly drifts (relaxing dynamics);

4) the relaxation is negligible for up-down strain steps.

Here, we propose a new model, henceforth called asymmetric power-law model (APL) for the reasons detailed in Appendix, able to compensate for these behaviors. The normalized version of model input $R$ and output $\hat{S}$ are $\xi=R / R_{0}+\alpha$ and $\psi=\hat{S} / S_{0}+\beta$, respectively, where parameters $\alpha, \beta, R_{0}$ and $S_{0}$ are chosen so that the dimensionless quantities $\xi$ and $\psi$ range in the interval $[-1,1]$.

The model is composed of a set of $N$ elementary hysteretic cells each one characterized by a state variable $x_{k}$ whose dynamics is defined through the implicit formulation $h_{k}\left(x_{k}, \dot{x}_{k}, t\right)=0, k=1, \ldots, N$. Functions $h_{k}$ are defined as follows:

$$
\begin{aligned}
& h_{k}\left(x_{k}, \dot{x}_{k}, t\right)= \\
& = \begin{cases}\dot{x}_{k}-\sigma\left(\frac{\xi(t)-x_{k}(t)+1}{\rho_{k}+1}\right)^{p} & \text { if }-1 \leq \xi(t)-x_{k} \leq \rho_{k} \\
x_{k}-\xi(t)-1 & \text { if } \dot{x}_{k} \leq 0 \\
x_{k}-\xi(t)+\rho_{k} & \text { if } \dot{x}_{k} \geq \sigma\end{cases}
\end{aligned}
$$

where $\rho_{k}=\frac{2 k}{N+1}-1(k=1, \ldots, N)$ are thresholds equally spaced in the range $(-1,1)$. Parameters $\sigma>0$ and $p>0$ determine the shape of the nonlinear vector field. In particular, $\dot{x}_{k}=\sigma$ for $\xi-x_{k}=\rho_{k}$, whereas $p$ determines the curvature of the vector field for $-1<\xi-x_{k}<\rho_{k}$ (e.g., for $p=1$ we obtain a straight line). A qualitative example of vector field is shown in Fig. 5 (left panel). 


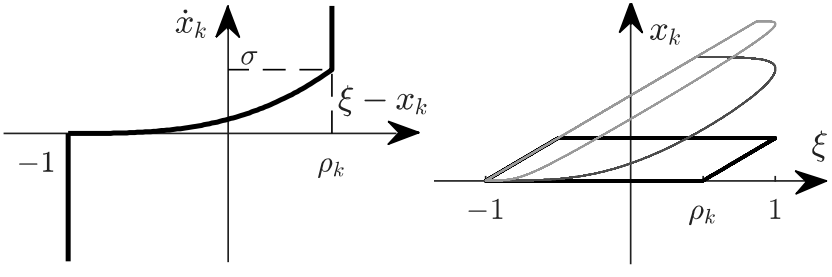

Fig. 5. Vector field (left panel) and hysteresis loops at different input rates (right panel) of the $k$-th cell of the APL model. In the left panel, curve colors become darker as the input rate increases.
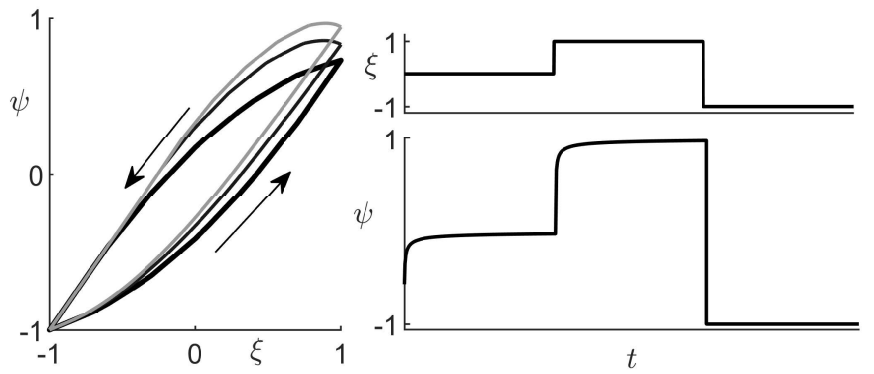

Fig. 6. Left panel: hysteresis loops $\psi$ vs $\xi$. Curve colors become darker as the input rate increases. Right panels: applied input (top) and APL model output (bottom).

The model output $\psi$ is computed as:

$$
\psi(t)=f^{-1}\left(w_{0} \xi+\sum_{k=1}^{N} w_{k} x_{k}+w_{N+1}\right)
$$

where $f$ is assumed to be a strictly-increasing piecewise-affine (PWA) function, defined as a weighted sum of an odd number $M$ of PWA basis-functions $\phi_{j}(j=1, \ldots, M)$ [29]:

$$
f(\psi)=\sum_{j=1}^{M} \mu_{j} \phi_{j}(\psi)
$$

Coefficients $w_{k}(k=0, \ldots, N+1)$ and $\mu_{j}(j=1, \ldots, M)$ are obtained by solving a quadratic programming problem based on experimental measurements of model inputs and outputs.

By applying a high-frequency triangular input $\xi$, the relationship between the state $x_{k}$ of the $k$-th APL cell and the input $\xi$ is the trapezoidal hysteresis loop shown in black in Fig. 5 (right panel). As the rate of the input increases, the loops are hinged at the lower-left corner. By summing states $x_{k}$ as in Eq. (2), it results (see Fig. 6, left panel) that the hysteresis loop between the model output $\psi$ and $\xi$ is traveled counterclockwise (see arrows) and rotates clockwise around its lower-left corner as input rate increases. This allows to compensate for the Electrolycra's nonlinearities. The right panels in Fig. 6 show instead the response (bottom panel) of the model to a stepwise input (top panel). Notice that the relaxation dynamics is exhibited only for increasing input steps.

Model parameters are $N, M, \sigma, p, w_{k}$, and $\mu_{j}$ and can be optimized with a procedure completely similar, mutatis mutandis, to the one proposed in [35] for the model of piezoelectric materials reported in Appendix for ease of comparison.
TABLE I

APL AND MPI MODEL PARAMETERS.

\begin{tabular}{r|c|c||c|c|} 
& \multicolumn{2}{|c||}{ APL model } & \multicolumn{2}{c|}{ MPI model } \\
\hline$k, j$ & $w_{k}\left(\times 10^{-6}\right)$ & $\mu_{j}\left(\times 10^{5}\right)$ & $w_{k}\left(\times 10^{-6}\right)$ & $\mu_{j}\left(\times 10^{5}\right)$ \\
\hline \hline 0 & 2.523 & & 1.483 & \\
1 & 0.843 & 0.412 & 0.510 & 0.518 \\
2 & 1.522 & 0.014 & 0.808 & -0.143 \\
3 & 0.522 & 0.092 & 0.337 & 0.295 \\
4 & 0.513 & 0.190 & 0.220 & 0.506 \\
5 & 0.002 & 1.405 & 0.233 & 2.516 \\
6 & 0.022 & 0.293 & 0.102 & 0.692 \\
7 & 0.041 & 0.056 & 0.070 & 0.131 \\
8 & 0.106 & 0.716 & 0.043 & 0.841 \\
9 & 0.276 & -0.160 & 0.038 & -0.367 \\
10 & 0.457 & & 0.068 & \\
11 & 0.695 & & 0.070 & \\
12 & 3.827 & & 0.026 & \\
\hline
\end{tabular}

\section{Results AND discussion}

We added the APL model to the MATLAB toolbox HysTool [36], which automatically fits its parameters to experimental data. We chose to use as training set the datasets SP1, SR1, SR4, SR7, SR9, whereas the other sets are used for validation purposes. The model inputs $\xi$ and outputs $\psi$ have been obtained by normalizing the values of $R$ and $S$ contained in the training set, with $R_{0}=7.16 \Omega, \alpha=-2.61, S_{0}=25 \%$ and $\beta=-1$. Through HysTool we set $N=11$ and $M=9$ (a further increase of their values does not decreases significantly the estimation error), $\sigma=2.15 \cdot 10^{-2}$ and $p=0.73$. The other fitting parameters (obtained by solving a quadratic problem) are listed in Tab. I (left columns).

HysTool has been also used to fit the MPI model (already implemented in the tool), in order to perform a comparison with the solution adopted in [37]. As shown in the Appendix, the MPI model relies on the same parameters of the APL model, with the exception of $\sigma$ and $p$. By setting a priori $N=11$ and $M=9$, the resulting optimal parameters are listed in Tab. I (right columns).

A third model (used as benchmark also in [37]) has been considered, which simply expresses the strain as a cubic (CU) function of the resistance, by neglecting the hysteresis:

$$
S=c_{3} R^{3}+c_{2} R^{2}+c_{1} R+c_{0}
$$

Coefficients $c_{0}, \ldots, c_{3}$ are obtained through least squares optimization on the considered data, leading to $c_{0}=-69.5, c_{1}=$ $7.816 \Omega^{-1}, c_{2}=-0.2112 \Omega^{-2}$ and $c_{3}=0.003512 \Omega^{-3}$.

Fig. 7 shows the root mean square error (RMSE) obtained with the APL, MPI and CU models on all the 13 available datasets, being $t_{k}(k=1, \ldots, K)$ the sampling times. The RMSE is defined as

$$
\mathrm{RMSE}=\sqrt{\frac{1}{K} \sum_{k=1}^{K}\left(\hat{S}\left(t_{k}\right)-S\left(t_{k}\right)\right)^{2}}
$$

As expected, the CU model leads to larger errors, since it does not represent hysteresis, whereas the APL model outperforms in most cases the MPI model, as it is able to reproduce ratedependent effects. In the following sections, more detailed results are shown for each class of datasets. 


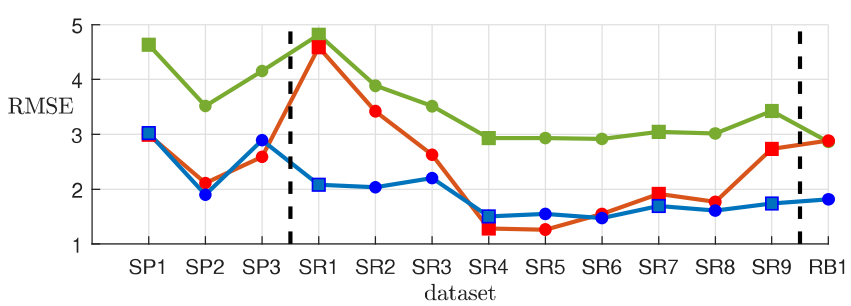

Fig. 7. RMSE obtained on all the 13 datasets employing APL model (blue curve), MPI model (red curve) and CU model (green curve). Squares mark the datasets used also to train the models.
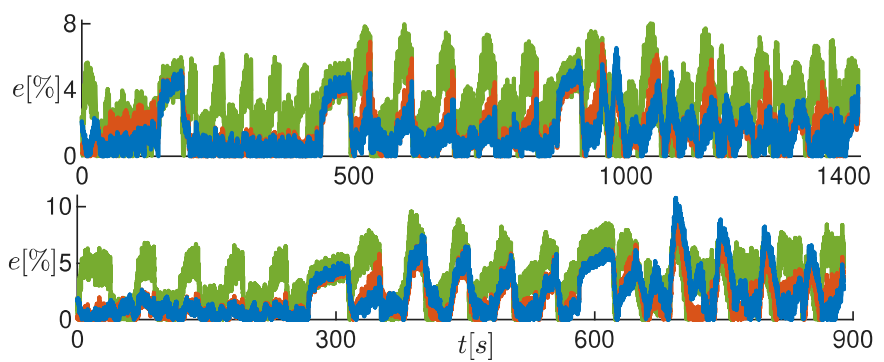

Fig. 8. Time evolution of $e(t)=|S(t)-\hat{S}(t)|$ for dataset SP2 (top panel) and SP3 (bottom panel) with APL (blue), MPI (orange) and CU (green) model.

\section{A. Different strain patterns}

On datasets SP1, SP2 and SP3 the performances of the APL and MPI models are comparable. Indeed, in these datasets the strain rate is constant, and therefore also the MPI model (which only models rate-independent hysteresis) is accurate. The absolute error $e(t)=|S(t)-\hat{S}(t)|$ as a function of time on datasets SP2 and SP3 (not used to train the model) is shown in Fig. 8, confirming that the accuracies of APL and MPI models are comparable and higher with respect to the $\mathrm{CU}$ model.

\section{B. Different strain rate}

The APL model, instead, outperforms the MPI model on datasets SR1-SR9, since the MPI model is not able to reproduce the rotation of the hysteresis loops as the strain rate increases. This is clearly visible in Fig. 9, which shows the time evolution of the measured and estimated strains (one period) for datasets SR2 (left) and SR9 (right). The bottom panels show the corresponding absolute errors. It can be noticed that the APL model is the only one able to correctly estimate the strain at both low and high rate with an error always lower than $4 \%$, as it reproduces the loop rotation, as visible in Fig. 10.

The ideal characteristic of the compensated sensor shown in Fig. 4 should be $\hat{S}=S$. Fig. 11 shows the characteristics obtained with the CU (left), MPI (center) and APL (right) models on datasets SR1, .., SR4. If the CU model is used, hysteresis is not compensated, as demonstrated by the loops in the $(S, \hat{S})$ plane. With the MPI model, the loop areas are greatly reduced, however the characteristics deviate from the ideal one (black dashed line) as the input rate decreases. On the contrary, by exploiting the APL model, hysteresis is well
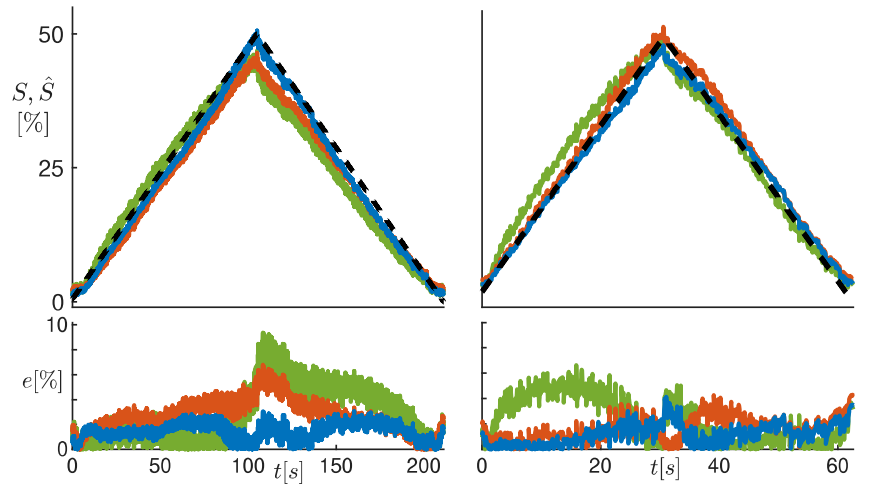

Fig. 9. Measured (black dashed curves) and estimated (color curves) strain $S$ for datasets SR2 (top left panel) and SR9 (top right panel). The bottom panels show the corresponding absolute errors. Blue curves: APL model; orange curves: MPI model; green curves: CU model.

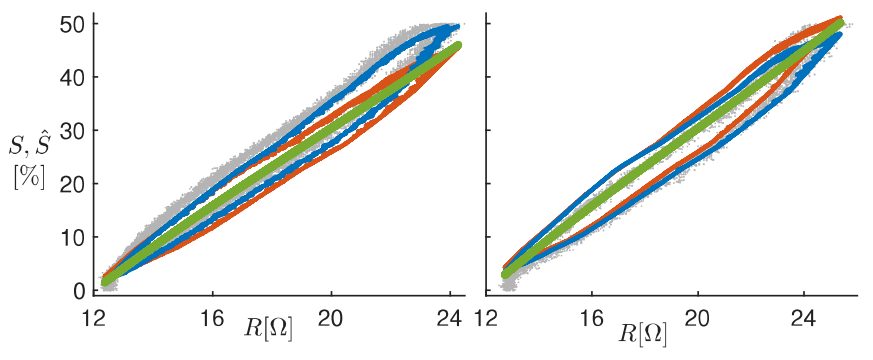

Fig. 10. Measured (gray points) and estimated (colored curves) strain $S$ vs. resistance $R$ for the lowest (SR1) and the highest (SR9) strain rates. Blue curve: APL model; orange curve: MPI model; green curve: CU model.

compensated and the characteristics remain close to the ideal one for all the considered input rates.

\section{Relaxation behavior}

For dataset RB1, the lowest error is achieved with the APL model because it is the only one able to reproduce the relaxation dynamics. This is also visible in Fig. 12 (see in particular the inset), where the APL model estimates an almost constant strain in response to a time decreasing resistance, meaning that the relaxation dynamics is at least partially compensated. In other steps the compensation is worse, especially in the first part of the steps, but anyway it is better than those obtained with the other considered models. We also remark that this dataset has not been used to identify the model parameters; this test is therefore a further proof of the generalization capability of the model.

\section{Conclusions}

A novel model (APL) has been proposed, able to reproduce the rate-dependent hysteresis of a piezoresistive textile (Electrolycra). The model has been derived from the powerlaw (PL) model, suitable for piezoelectric actuators, which also exhibit rate-dependent hysteresis, but with remarkable differences, as pointed out in the Appendix.

Different from [25] and [37], where only few tests are shown, the APL model has been validated on 13 datasets 

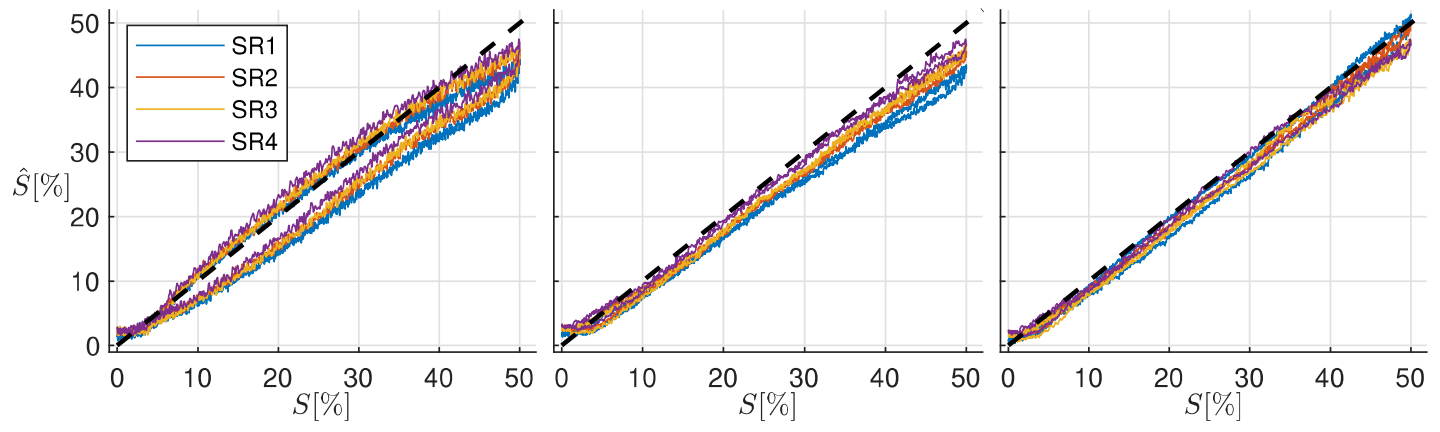

Fig. 11. Estimated strain $\hat{S}$ with CU (left panel), MPI (middle panel) and APL (right panel) model as a function of the measured strain $S$. The black dashed line represents the ideal characteristic $\hat{S}=S$.

(only 5 of them have been exploited to identify the model parameters) comprising measurements of strain and resistance, in three completely different scenarios. Analogously to the PL model, also the APL model is suitable for implementation on digital devices (e.g., microcontrollers [38]), making it suitable for a real-time estimation of the textile strain, given the measurement of its electrical resistance. This allows to develop accurate strain sensors based on piezoresistive textile, suitable for soft robotics applications.

This work also adds relevant results to the wearable technologies field. In particular, a correct estimation of the strain for wearable flexible sensors is of a paramount important for joint angle measurements, posture monitoring, motion patterns recognition, and so forth. For this reason, it is expected to be especially useful for real-time continuous measurement without influencing the wearer?s activity, following the "wear and forget" concept. Among the other characteristics, this strain sensor based on piezoresistive textile is non-invasive, easy to use, inexpensive and it offers the possibility of measurements outside the laboratory setting.

The APL model provided a good hysteresis compensation performance even with a small calibration dataset. These results confirm that an adequate processing can enhance the sensor performances in case of dynamic measurements, without a prior knowledge of the strain rate typical for a particular application, extending its general applicability. Moreover, since hysteresis is caused by the intrinsic mechanical properties of the elastic material (that requires longer recovery time) the approach can be extended to all the sensors which are based on elastomer deformations and present rate-dependent hysteresis to improve their estimation performance.

\section{APPENDIX}

The proposed APL model has been conceived starting from the power-law (PL) hysteresis and creep model of piezoelectric actuators (PEAs) proposed in [29], [35]. In this Appendix we point out similarities and differences between APL and PL model.

PL model is tailored for PEAs, i.e., actuators that exhibit a displacement $z$ when a certain voltage $V$ is applied, which are largely used for micro- and nano-positioning applications [30], [31]. In general, the relationship between $z$ and $V$ in PEAs is a hysteresis loop that rotates around its center when the input

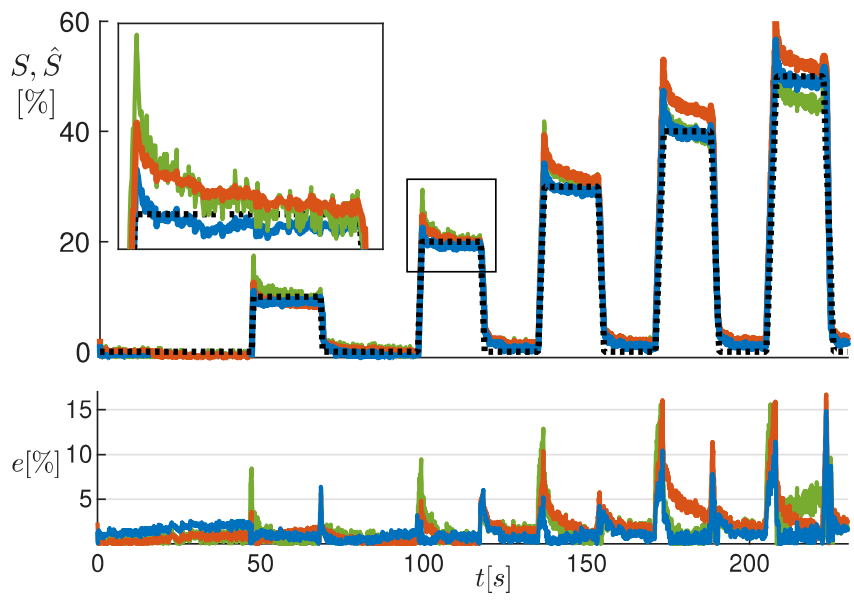

Fig. 12. Measured (black dashed lines) and estimated (color curves) strain $S$ computed on dataset RB1. Blue curve: APL model; orange curve: MPI model; green curve: CU model.

rate changes, as shown in Fig. 13. The creep effect is instead a slow elongation or contraction, in response to a piecewiseconstant applied voltage [38]. The following analogies and differences between PEAs and Electrolycra can be observed:

1) the relationship between $z$ and $V$ for PEAs and $S$ and $R$ for Electrolycra is hysteretical, and the hysteresis loop is traveled counterclockwise;

2) in PEAs the hysteresis loop rotates clockwise around its center as the voltage rate increases, whereas in the Electrolycra it rotates clockwise around its lower-left corner as the strain rate increases;

3 ) in the presence of a step variation of voltage, the PEA deformation slowly drifts (creep effect); the same happens for the resistance of the Electrolycra in response to a strain step (relaxing dynamics); ${ }^{1}$

4) in PEAs the creep is visible for both up-down and downup voltage steps [38] (see right panel of Fig. 14 for a qualitatively description), whereas in the Electrolycra the relaxation is negligible for up-down strain steps (see Fig. 3).

${ }^{1}$ Notice that for PEAs the direction of the relaxation dynamics is coherent with the direction of the step [38], which is not the case for Electrolycra (see Fig. 3). However, in the PL model the deformation is the output, whereas in the APL model the resistance is the input. 


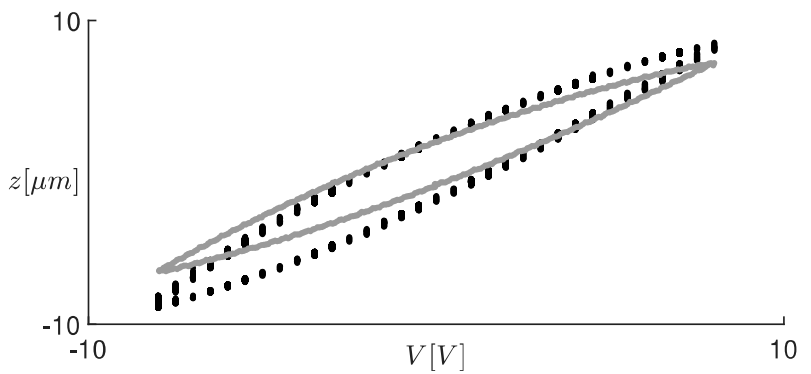

Fig. 13. Experimental loops obtained on a PEA with low (black dots) and high (gray dots) input rate.

The above analogies suggested us that the PL model could be suitable also to reproduce the nonlinear dynamical behaviors of the Electrolycra, after some modifications.

The APL model differs from the PL model proposed in [29] in the definition of implicit function $h_{k}\left(x_{k}, \dot{x}_{k}, t\right)$. For the PL model it is defined as:

$$
h_{k}\left(x_{k}, \dot{x}_{k}, t\right)=\left\{\begin{array}{c}
\dot{x}_{k}-\sigma\left(\frac{\left|\xi(t)-x_{k}(t)\right|}{\rho_{k}}\right)^{p} \cdot \operatorname{sgn}\left(\xi(t)-x_{k}(t)\right), \\
\text { if }\left|x_{k}-\xi(t)\right| \leq \rho_{k} \\
x_{k}-\xi(t)-\rho_{k}, \text { if } \dot{x}_{k} \leq-\sigma \\
x_{k}-\xi(t)+\rho_{k}, \text { if } \dot{x}_{k} \geq \sigma
\end{array}\right.
$$

where $\rho_{k}=\frac{k}{N+1}(k=1, \ldots, N)$ are thresholds equally spaced in the range $(0,1)$ and function $\operatorname{sgn}(\cdot)$ returns 1 if its argument is positive and -1 otherwise. The corresponding nonlinear vector field is shown in Fig. 14 (the name "powerlaw" derives from its central region) and its shape is determined by hyper-parameters $\sigma>0$ and $p>0$ which, together with $N$ and $M$ must be set a priori [35]. The PL model reduces to the well-known MPI model for $\sigma=0$ (in this case, $p$ has no influence). By applying a triangular input $\xi$, the relationship between the state $x_{k}$ of the $k$-th PL cell and the input $\xi$ is the trapezoidal hysteresis loop shown in black in Fig. 14 (right panel). As the input frequency decreases, the loop becomes thinner, higher and smoother, but it maintains its central symmetry. By contrast, in the APL model, when the input rate decreases the loops rotate around the lowerleft corner, due to the different shape of the vector field. By summing states $x_{k}$ obtained with the PL model as in Eq. (2), it results that the hysteresis loop between the model output $\psi$ and $\xi$ is traveled counterclockwise and rotates clockwise around its center (Fig. 15, left panel) as the input frequency increases. The right panels in Fig. 15 show instead the response (bottom panel) of the model to a step-wise input (top panel). Notice that, differently from the APL model, the relaxation dynamics is exhibited both for increasing and decreasing input steps.

\section{REFERENCES}

[1] V. Feliu and F. Ramos, "Strain gauge based control of single-link flexible very lightweight robots robust to payload changes," Mechatronics, vol. 15 , no. 5, pp. 547-571, 2005.

[2] M. C. Carrozza, A. Eisinberg, A. Menciassi, D. Campolo, S. Micera, and P. Dario, "Towards a force-controlled microgripper for assembling biomedical microdevices," J. Micromech. Microeng., vol. 10, no. 2, p. 271,2000

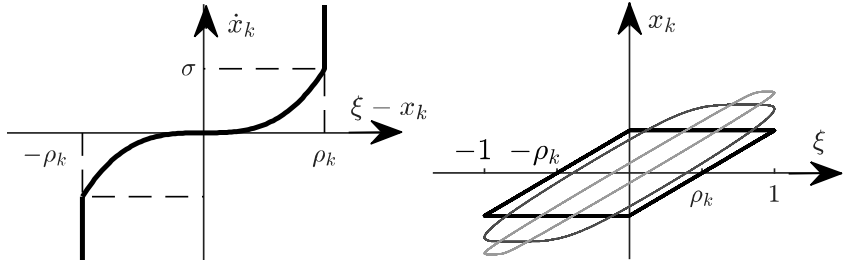

Fig. 14. Vector field (left panel) and hysteresis loops at different input rates (right panel) of the $k$-th cell of the PL model. In the left panel, curve colors become darker as the input rate increases.
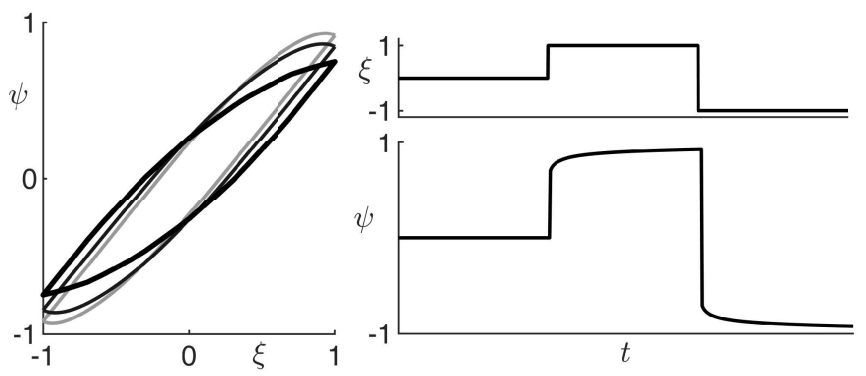

Fig. 15. Left panel: hysteresis loops $\psi$ vs $\xi$. Curve colors become darker as the input rate increases. Right panels: applied input (top) and PL model output (bottom).

[3] J.-B. Chossat, Y.-L. Park, R. J. Wood, and V. Duchaine, "A soft strain sensor based on ionic and metal liquids," IEEE Sensors J., vol. 13, no. 9, pp. 3405-3414, 2013.

[4] T. Yamada, Y. Hayamizu, Y. Yamamoto, Y. Yomogida, A. IzadiNajafabadi, D. N. Futaba, and K. Hata, "A stretchable carbon nanotube strain sensor for human-motion detection," Nature nanotechnol., vol. 6, no. 5 , p. $296,2011$.

[5] D.-H. Kim, R. Ghaffari, N. Lu, and J. A. Rogers, "Flexible and stretchable electronics for biointegrated devices," Ann. rev. biom. eng., vol. 14, pp. 113-128, 2012

[6] Y. Mengüç, Y.-L. Park, H. Pei, D. Vogt, P. M. Aubin, E. Winchell, L. Fluke, L. Stirling, R. J. Wood, and C. J. Walsh, "Wearable soft sensing suit for human gait measurement," Int. J. Rob. Res., vol. 33, no. 14, pp. $1748-1764,2014$

[7] P. Polygerinos, Z. Wang, K. C. Galloway, R. J. Wood, and C. J. Walsh, "Soft robotic glove for combined assistance and at-home rehabilitation," Robotics Auton. Syst., vol. 73, pp. 135-143, 2015.

[8] M. Cianchetti, F. Renda, A. Licofonte, and C. Laschi, "Sensorization of continuum soft robots for reconstructing their spatial configuration," in 4th IEEE RAS \& EMBS Int. Conf. on Biomedical Robotics and Biomechatronics (BioRob), 2012, pp. 634-639.

[9] P. Polygerinos, N. Correll, S. A. Morin, B. Mosadegh, C. D. Onal, K. Petersen, M. Cianchetti, M. T. Tolley, and R. F. Shepherd, "Soft robotics: Review of fluid-driven intrinsically soft devices; manufacturing, sensing, control, and applications in human-robot interaction," Adv. Eng. Mat., vol. 19, no. 12, p. 1700016, 2017.

[10] K. J. Loh, J. P. Lynch, B. Shim, and N. Kotov, "Tailoring piezoresistive sensitivity of multilayer carbon nanotube composite strain sensors," $J$. Intell. Material Syst. Struct., vol. 19, no. 7, pp. 747-764, 2008.

[11] O. K. Johnson, C. J. Gardner, D. T. Fullwood, B. L. Adams, N. Hansen, and G. Hansen, "The colossal piezoresistive effect in nickel nanostrand polymer composites and a quantum tunneling model," Computers, Materials, \& Continua, vol. 15, no. 2, pp. 87-112, 2010.

[12] Y.-L. Park, B.-R. Chen, and R. J. Wood, "Design and fabrication of soft artificial skin using embedded microchannels and liquid conductors," IEEE Sensors J., vol. 12, no. 8, pp. 2711-2718, 2012.

[13] J. T. Muth, D. M. Vogt, R. L. Truby, Y. Mengüç, D. B. Kolesky, R. J. Wood, and J. A. Lewis, "Embedded 3d printing of strain sensors within highly stretchable elastomers," Adv. Materials, vol. 26, no. 36, pp. 63076312, 2014

[14] A. Frutiger, J. T. Muth, D. M. Vogt, Y. Mengüç, A. Campo, A. D. Valentine, C. J. Walsh, and J. A. Lewis, "Capacitive soft strain sensors via multicore-shell fiber printing," Adv. Materials, vol. 27, no. 15, pp. $2440-2446,2015$ 
[15] M. Kollosche, H. Stoyanov, S. Laflamme, and G. Kofod, "Strongly enhanced sensitivity in elastic capacitive strain sensors," J. Materials Chem., vol. 21, no. 23, pp. 8292-8294, 2011.

[16] A. Morteza, K. Ki-Uk, P. Inkyu, and S. Metin, "Stretchable, skinmountable, and wearable strain sensors and their potential applications: A review," Adv. Funct. Materials, vol. 26, no. 11, pp. 1678-1698, 2016.

[17] F. Lorussi, N. Carbonaro, D. De Rossi, and A. Tognetti, "Strain- and Angular-Sensing Fabrics for Human Motion Analysis in Daily Life," in Smart Textiles: Fundamentals, Design, and Interaction, pp. 49-70. Cham: Springer International Publishing, 2017.

[18] L. M. Castano and A. B. Flatau, "Smart fabric sensors and e-textile technologies: a review," Smart Materials Struct., vol. 23, no. 5, p. 053001, 2014.

[19] P. Bahrami, N. Yamamoto, Y. Chen, and H. Manohara, "Capacitancebased damage detection sensing for aerospace structural composites," in Sensors and Smart Structures Technologies for Civil, Mechanical, and Aerospace Systems 2014, vol. 9061. United States: SPIE, 2014.

[20] M. Sergio, N. Manaresi, M. Nicolini, D. Gennaretti, M. Tartagni, and R. Guerrieri, "A textile-based capacitive pressure sensor," Sensor Lett., vol. 2, no. 2, pp. 153-160, 2004

[21] V.-G. Hardy, "Design and construction of smart structures for technical textiles," Ph.D. dissertation, University of Leeds, 2008.

[22] J. Fraden, Handbook of modern sensors: physics, designs, and applications. Springer Science \& Business Media, 2004.

[23] L. Wang, Y. Han, C. Wu, and Y. Huang, "A solution to reduce the time dependence of the output resistance of a viscoelastic and piezoresistive element," Smart Materials Struct., vol. 22, no. 7, p. 075021, 2013.

[24] O. Atalay and W. R. Kennon, "Knitted strain sensors: Impact of design parameters on sensing properties," Sensors, vol. 14, no. 3, pp. 47124730, 2014.

[25] M. R. Sunny and R. K. Kapania, "A hysteresis compensator based on a modified dynamic preisach model for conductive polymer nanocomposites," in Proc. of IUTAM Symp. on Multi-Functional Material Structures and Systems, pp. 87-94. Dordrecht: Springer Netherlands, 2010.

[26] J.-S. Kim and G.-W. Kim, "Hysteresis compensation of piezoresistive carbon nanotube/polydimethylsiloxane composite-based force sensors," Sensors, vol. 17, no. 2, p. 229 (1-12), 2017

[27] V. Hassani, T. Tjahjowidodo, and T. N. Do, "A survey on hysteresis modeling, identification and control," Mechanical systems and signal processing, vol. 49, no. 1-2, pp. 209-233, 2014.

[28] G.-Y. Gu, L.-M. Zhu, C.-Y. Su, H. Ding, and S. Fatikow, "Modeling and control of piezo-actuated nanopositioning stages: A survey." IEEE Trans. Autom. Sci. Eng., vol. 13, no. 1, pp. 313-332, 2016.

[29] M. Biggio, A. Oliveri, F. Stellino, M. Parodi, and M. Storace, "A circuit model of hysteresis and creep," IEEE Trans. Circuits Syst. II, Exp. Briefs, vol. 62 , no. 5, pp. 501-505, 2015.

[30] S. Devasia, E. Eleftheriou, and S. R. Moheimani, "A survey of control issues in nanopositioning," IEEE Trans. Control Syst. Technol., vol. 15, no. 5 , pp. $802-823,2007$

[31] Z. Sun, B. Song, N. Xi, R. Yang, L. Hao, Y. Yang, and L. Chen, "Asymmetric hysteresis modeling and compensation approach for nanomanipulation system motion control considering working-range effect," IEEE Trans. Ind. Electron., vol. 64, no. 7, pp. 5513-5523, 2017.

[32] C. Lucarotti, M. Totaro, A. Sadeghi, B. Mazzolai, and L. Beccai, "Revealing bending and force in a soft body through a plant root inspired approach," Scient. Reports, vol. 5, p. 8788, 2015.

[33] A. Grassi, F. Cecchi, M. Maselli, M. Röling, C. Laschi, and M. Cianchetti, "Warp-knitted textile as a strain sensor: Characterization procedure and application in a comfortable wearable goniometer," IEEE Sensors J., vol. 17, no. 18, pp. 5927-5936, Sep. 2017.

[34] M. Maselli, E. Mussi, F. Cecchi, M. Manti, P. Tropea, and C. Laschi, "A wearable sensing device for monitoring single planes neck movements: assessment of its performance," IEEE Sensors J., vol. 18, no. 15, pp. 6327-6336, 2018.

[35] A. Oliveri, M. Lodi, M. Parodi, F. Stellino, and M. Storace, "Model reduction for optimized online compensation of hysteresis and creep in piezoelectric actuators," IEEE Trans. Circuits Syst. II, Exp. Briefs, pp. $1-1,2017$.

[36] A. Oliveri, M. Lodi, F. Stellino, and M. Storace, "Modeling and compensation of hysteresis and creep: The HysTool toolbox," in Proc. of the 2018 IEEE Int. Symp. on Circuits and Systems (ISCAS), , pp. 1-5.

[37] H. Lee, H. Cho, S. J. Kim, Y. Kim, and J. Kim, "Dispenser printing of piezo-resistive nanocomposite on woven elastic fabric and hysteresis compensation for skin-mountable stretch sensing," Smart Materials Struct., vol. 27, no. 2, p. 025017, 2018.
[38] A. Oliveri, F. Stellino, G. Caluori, M. Parodi, and M. Storace, "Openloop compensation of hysteresis and creep through a power-law circuit model," IEEE Trans. Circuits Syst. I, Reg. Papers, vol. 63, no. 3, pp. 413-422, Mar. 2016.

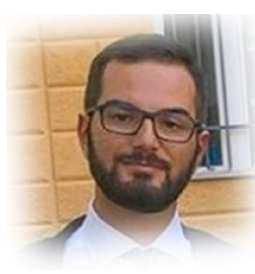

Alberto Oliveri (M'17) received the M.Sc. and $\mathrm{Ph}$.D. degrees in electrical engineering from the University of Genoa, Genova, Italy, in September 2009 and April 2013, respectively.

$\mathrm{He}$ is currently an Assistant Professor in the Department of Electrical, Electronic, Telecommunications Engineering, and Naval Architecture, University of Genoa.

Dr. Oliveri's main research interests include the design and circuit implementation of explicit exact and approximate model predictive control functions and piecewise-affine virtual sensors. He currently serves as Associate Editor for the IEEE Transactions on Circuits and Systems I.

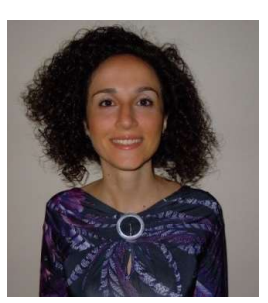

Martina Maselli received the B.Sc. and M.Sc. (Summa Cum Laude) degrees in Biomedical Engineering from the University of Pisa, in 2012 and 2015 , respectively. She is currently pursuing the Ph.D. degree in BioRobotics with the Scuola Superiore Sant?Anna, Pisa. Her main research interests include study and improvement of concepts and applications of textile sensors for pressure and strain detection.

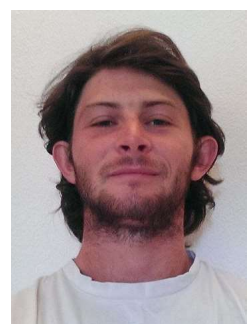

Matteo Lodi (S'18) was born in Genoa, Italy, in 1991. He received the "Laurea" (M.Sc.) five-year degree (Summa Cum Laude) in Electronic Engineering in 2015 and is currently working towards his Ph.D. degree in Electrical Engineering at the University of Genoa, Italy. He was a visitor to Georgia State University, Atlanta, USA, in 2016. His main research interests are in the area of modeling of nonlinear systems (hysteresis and networks of biological neurons), bifurcation analysis and nonlinear dynamics.

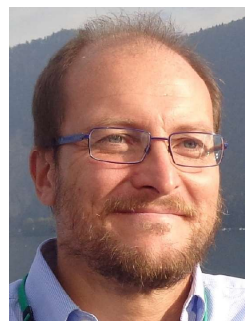

Marco Storace (M'01, SM'14) was born in Genoa, Italy, in 1969. He received the "Laurea" (M.Sc.) five-year degree (Summa Cum Laude) in Electronic Engineering in March 1994 and the Ph.D. degree in Electrical Engineering in April 1998, both from the University of Genoa, Italy. Since 2011 he has been a Full Professor in the University of Genoa. He was a visitor to EPFL, Lausanne, Switzerland, in 1998 and 2002. His main research interests are in the area of nonlinear circuit theory and applications, with emphasis on models of nonlinear systems (e.g., hysteresis, biological neurons), methods for the piecewise-linear approximation of nonlinear systems and for the consequent circuit synthesis, bifurcation analysis and nonlinear dynamics. He is the author or coauthor of more than 140 scientific papers, more than an half of which have been published in international journals. Prof. Storace served as Associate Editor of the IEEE TRANSACTIONS ON CIRCUITS AND SYSTEMS - II (20082009) and is currently Secretary of the IEEE Technical Committee on Nonlinear Circuits and Systems (TC-NCAS).

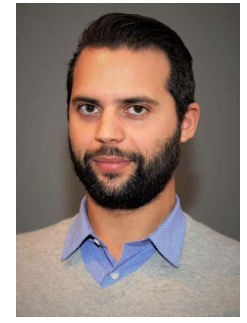

Matteo Cianchetti is currently Assistant Professor with The BioRobotics Institute of Scuola Superiore Sant'Anna (SSSA). He received the MSc degree in Biomedical Engineering (cum laude) from the University of Pisa, Italy, in 2007 and the $\mathrm{PhD}$ degree in Biorobotics (cum laude) from SSSA. He is author or co-author of more than 40 international peer reviewed papers and he regularly serves as a reviewer for more than 10 international ISI journals. He has been and currently is involved in several EU-funded projects with the main focus on the development of Soft Robotics technologies (including flexible fluidic actuators, granular jamming based systems, electro-active polymers and shape memory alloys). His main research interests include the study and development of new systems and technologies based on soft/flexible materials for soft actuators, smart compliant sensors and flexible mechanisms. 